\title{
Total parenteral nutrition associated cholestasis: a predisposing factor for sepsis in surgical neonates?
}

\author{
A.P.Bos, D.Tibboel, F. W.J.Hazebroek, J.H. Bergmeijer, E.J. van Kalsbeek, and J. C.Molenaar \\ Paediatric Surgical Intensive Care Unit, Department of Paediatric Surgery, Sophia Children's Hospital, Erasmus University Medical School, \\ Gordelweg 160, 3038 GE Rotterdam, The Netherlands
}

\begin{abstract}
Of 496 neonates and infants less than 1 year of age admitted to the paediatric surgical intensive care unit (PSICU) over a 5 year period (1983-1987), 94 required total parenteral nutrition (TPN) for more than 14 consecutive days, generally due to congenital anomalies of the digestive tract. Cholestasis occurred in 15 of them and 12 of these patients developed sepsis. In contrast, of the 79 patients on TPN that remained free from cholestasis, only 23 developed sepsis. The mortality rate for the TPNAC-group was substantially higher than for the group without TPNAC. It is suggested that development of TPNAC might lead to impairment of non-specific cellular immunity in neonates.
\end{abstract}

Key words: Cholestasis - Immune system - Neonatal - Septicaemia - Total parenteral nutrition

\section{Introduction}

Survival of neonates with congenital anomalies such as abdominal wall defects, intestinal atresia, necrotizing enterocolitis and short bowel syndrome has increased in the last decades. The administration of prolonged total parenteral nutrition (TPN) has become standard procedure in the management of these patients. Cholestasis is one of many complications that have been associated with TPN [6]. The aetiology of TPN-associated cholestasis (TPNAC) is unknown, but prematurity, low birth weight, the administration of amino acids and lack of enteral feeding have all been implicated as contributing factors [12]. Analysing the incidence of sepsis in our paediatric surgical intensive care unit (PSICU), we found a high frequency in TPNAC patients. This suggests that the development of cholestasis in patients who require prolonged TPN might be a predisposing factor for the occurrence of sepsis. In order to evaluate this premise, we carried out a retrospective investigation of sepsis in patients that received prolonged TPN and developed cholestasis.

Offprint requests to: A.P.Bos

Abbreviations: PSICU = pediatric surgical intensive care unit; TPN $=$ total parenteral nutrition; TPNAC $=$ total parenteral nutrition associated cholestasis;

\section{Patients and methods}

From January 1983 to December 1987, a total of 496 infants with congenital anomalies were admitted within 1 month after birth to the PSICU of Sophia Children's Hospital, Rotterdam. The patients that had received TPN for more than 14 consecutive days were considered for a retrospective evaluation. Excluding patients with congenital anomalies of the liver, such as biliary atresia, or hepatitis, and those who developed sepsis within the first 14 days of TPN, the study population finally consisted of 94 patients.

The perinatal parameters for these 94 patients were assessed, such as duration of gestation, birth weight, gender, morbidity and mortality and diagnosis at admission. Depending on the occurrence of cholestasis, these patients were assigned to either one of two groups, with the diagnosis of TPNAC defined by the following criteria:

1. The administration of TPN for at least 14 consecutive days.

2. A conjugated bilirubin level of $1.5 \mathrm{mg} / \mathrm{dl}$ or higher.

3. At least $50 \%$ of the total serum bilirubin in the direct reacting fraction.

4. Other problems associated with direct hyperbilirubinaemia, were ruled out either by analysis of serum or urine (e.g. infections, alpha-1-antitrypsin deficiency, galactosaemia) or ultrasound (malformation of bile ducts).

The nutritional regimen for all patients included amino acids (Amino-venös, Fresenius AG, Bad Homburg, FRG), fat (Intra lipid, Kabi-Vitrum, Stockholm, Sweden) and dextrose providing a daily intake of $100-120 \mathrm{kcal} / \mathrm{kg}, 2-2.5 \mathrm{~g} / \mathrm{kg}$ of protein and $2-3 \mathrm{~g} / \mathrm{kg}$ of fat. Vitamins and trace-elements (Pedel, Kabi-Vitrum, Stockholm, Sweden) were also administered.

The development of sepsis was noted per group. In cases of clinical suspicion of sepsis, septic episodes were only taken into account if substantiated by positive cultures of blood, CSF, urine, or central venous catheters. Data analysis included chi-square analysis of categorical data. The Student's $t$ test was used for comparison of mean data. Statistical significance was based on $P<0.05$.

\section{Results}

Of the 94 patients under study, $15(16 \%)$ developed TPNAC, while $79(84 \%)$ remained free from cholestasis. In both groups 
Table 1. Clinical data

\begin{tabular}{llll}
\hline & $\begin{array}{l}\text { Patients } \\
\text { with TPNAC }\end{array}$ & $\begin{array}{l}\text { Patients } \\
\text { without TPNAC }\end{array}$ & $P$ \\
\hline $\begin{array}{l}\text { Patients } \\
\begin{array}{l}\text { Duration } \\
\text { of gestation (week) }\end{array}\end{array}$ & 79 & \\
$\quad$ Mean & 36.5 & 36.5 & NS \\
$\quad$ Range & $31-40$ & $27-42$ & \\
$\begin{array}{l}\text { Birth weight (g) } \\
\quad \text { Mean }\end{array}$ & 2600 & 2450 & NS \\
$\quad$ Range & $2300-3965$ & $1000-4030$ & \\
Gender & & & NS \\
$\quad$ Male & 11 & 42 & \\
$\quad$ Female & 4 & 37 & 0.006 \\
Sepsis & $12(80 \%)$ & $23(29 \%)$ & 0.009 \\
Mortality & $3(20 \%)$ & $1(1.3 \%)$ & \\
\hline
\end{tabular}

Table 2. Characteristics of sepsis

\begin{tabular}{lll}
\hline & $\begin{array}{l}\text { Patients } \\
\text { with } \\
\text { TPNAC }\end{array}$ & $\begin{array}{l}\text { Patients } \\
\text { without } \\
\text { TPNAC }\end{array}$ \\
\hline Number of patients & 15 & 79 \\
Patients with sepsis & $12(80 \%)$ & $23(29 \%)$ \\
Number of septic episodes & 16 & 25 \\
Gram-positive organisms & 5 & 10 \\
S. epidermidis & 3 & 8 \\
S. aureus & 2 & 2 \\
Gram-negative organisms & 8 & 8 \\
Klebs. pneumoniae & 5 & 4 \\
Pseud. aeruginosa & 2 & 1 \\
E. coli & 1 & 3 \\
Other organisms & 3 & 7 \\
\hline
\end{tabular}

the incidence of abdominal wall defects, intestinal atresia and NEC was equally distributed. Table 1 gives the comparative clinical data for the two groups. There was a significant difference in the frequency of sepsis between the two groups, amounting to $80 \%$ (12 of 15 patients) for the TPNAC group versus $29 \%$ ( 23 of 79 patients) for the other group $(P=0.006)$. In the TPNAC group sepsis occurred 10-15 days (median 13 days) after cholestasis had developed. The mortality rate for the TPNAC group was $20 \%$, significantly higher than the $1.3 \%$ for the other group $(P=0.009)$. Table 2 gives the characteristics of sepsis as it occurred in either group. In the TPNAC group, 12 patients $(80 \%)$ developed sepsis, with the number of septic episodes amounting to 16 . In comparison, 23 (29\%) of the patients without cholestasis developed sepsis, amounting to 25 septic episodes. In the TPNAC group, the majority of the septic episodes were due to gram-negative organisms, while gram-positive organisms predominated in the other group. Statistical significance was not achieved in either group. The three remaining septic episodes in the TPNAC group were due to Candida albicans. In the group without TPNAC, seven septic episodes were due to other organisms, mainly consisting of urinary tract infection caused by Enterococcus. Sepsis-related mortality in the TPNAC group was caused by Pseudomonas aeruginosa and Staphylococcus aureus. The one death in the group without TPNAC was also related to sepsis, which was caused by Staphylococcus epidermidis.

\section{Discussion}

Long-term TPN has become a lifesaving treatment for neonates with surgical anomalies that compromise intestinal function. Peden and coworkers first reported the occurrence of cholestatic liver disease in a neonate who died following prolonged TPN [6]. Fifteen of our series of 94 patients who received TPN for over 14 consecutive days, developed cholestasis. This represents an incidence of $16 \%$, a figure that compares favourably with an incidence of approximately $30 \%$ reported in the literature $[4,7]$. The pathogenesis of the TPNAC syndrome is considered to be multifactorial [12]. Prematurity, low birth weight and amino acid components of TPN, in combination with a lack of enteral feeding have all been associated with the syndrome [2]. In our study, clinical characteristics associated with the development of TPNAC, such as low birth weight and prematurity, failed to achieve statistical significance.

Sepsis per se can be an aetiological factor for the development of cholestasis [12]. Therefore we excluded the patients from study, who became septic during the first 14 days of TPN. After TPNAC had occurred, $80 \%$ of the patients developed sepsis, compared with $29 \%$ without cholestasis. This difference was not related to the number of indwelling central venous catheters in either group.

Gram-negative infections predominated in the TPNACgroup and were responsible for fatal septic episodes in two patients. Yeast septicaemia contributed to a prolonged stay in the PSICU for three other patients.

Little is known of the effect of cholestasis on host immunity. Recent review articles in the paediatric literature on neonatal cholestasis [1] and neonatal immunity [13] do not dwell on this aspect. However, in 1975 Rola-Pleszczynski et al. described inhibitory effects of bilirubin on cell-mediated immunity as measured by Imyphoproliferative responses to phytohaemagglutinin in adult and cord blood lymphocytes [8]. Experimental biliary obstruction in rats showed impairment of both in vitro and in vivo specific cellmediated immunity. This impairment was partly reversible by internal biliary drainage [9]. Recently, delayed hypersensitivity was observed in jaundiced, adult patients, confirming depression of cell-mediated immunity in these patients [3]. Further investigation of nonspecific cellular immune function in a rat model of bile duct ligation showed impairment of phagocytic ability of polymorphonuclear leukocytes as well as pulmonary alveolar macrophages. Direct migration and superoxide release were not affected [10].

We suggest that also in neonates impairment of non-specific, cellular immunological mechanisms may occur in association with cholestasis and would account for the increased susceptibility to sepsis in TPNAC patients. One of the essential processes that must take place prior to phagocytosis, is opsonization of bacteria which is partly dependent on the presence of complement and fibronectin. One could only speculate whether fibronectin deficiency plays a role in this patient group, although fibronectin deficiency already has been related to sepsis in adults $[5,11]$. 
Acknowledgements. The authors wish to thank Mrs. A. M. C. RibbinkGoslinga for editing the manuscript and Mrs. E. van der Ham for secretarial assistance.

\section{References}

1. Balistreri WF (1985) Neonatal cholestasis. J Pediatr 106:171-182

2. Beale EF, Nelson RM, Bucciarelli RL, Donnelly WH, Eitzman DV (1979) Intrahepatic cholestasis associated with parenteral nutrition in premature infants. Pediatrics $64: 342-346$

3. Cainzos M, Potel J, Puente JL (1988) Anergy in jaundiced patients. Br J Surg 75:147-149

4. Ginn-Pease ME, Pantalos D, King R (1985) TPN-associated hyperbilirubinemia: a common problem in newborn surgical patients. J Pediatr Surg 20:436-439

5. Hesselvik JF (1987) Plasma fibronectin levels in sepsis: influencing factors. Crit Care Med 15:1092-1097

6. Peden VH, Witzleben DL, Stelton MA (1971) Total parenteral nutrition. J Pediatr 78:180-183
7. Postuma R, Trevenen CL (1979) Liver disease in infants receiving total parenteral nutrition. Pediatrics $63: 110-115$

8. Rola-Pleszczynski M, Hensen SA, Vincent MM, Bellanti JA (1975) Inhibitory effects of bilirubin on cellular immune responses in man. J Pediatr 86:690-696

9. Roughneen PT, Gouma DJ, Kulkarni AD, Fanslow WF, Rowlands BJ (1986) Impaired specific cell-mediated immunity in experimental biliary obstruction and its reversibility by internal biliary drainage. J Surg Res 41:113-125

10. Roughneen PT, Drath DB, Kulkarni AD, Rowlands BJ (1987) Impaired nonspecific cellular immunity in experimental cholestasis. Ann Surg 206:578-582

11. Saba TM, Kiener JL, Holman JM (1986) Fibronectin and the critically ill patient: current status. Intensive Care Med 12:350-358

12. Sinatra FR (1982) Cholestasis in infancy and childhood. Curr Probl Pediatr 12:33

13. Wilson CB (1986) Immunologic basis for increased susceptibility of the neonate to infection. J Pediatr 108:1-12

Received October 10,1988 / Accepted May 10, 1989 